\title{
The Effect of Ankle Joint Muscle Strengthening Training and Static Muscle Stretching Training on Stroke Patients' C.O.P Sway Amplitude
}

\author{
Tae Ho Kim, PhD ${ }^{1)}$, Joo Soo Yoon, MS, PT ${ }^{1)}$, Jin Hwan Lee, MS, PT ${ }^{1)^{*}}$ \\ 1) Department of Physical Therapy, College of Rehabilitation Science, Daegu University: 15 Jilyang, \\ Gyeongsan-si, Kyeongbuk 712-714, Republic of Korea
}

\begin{abstract}
Purpose] This study implement ankle joint dorsiflexion training for ankle muscle the weakness that impairs stroke patients' gait performance, to examine the effect of the training on stroke patients' plantar pressure and gait ability. [Subjects and Methods] In this study, 36 stroke patients diagnosed with stroke due to cerebral infarction or cerebral hemorrhage performed the training. Static muscle stretching was performed four times a week for 20 minutes at a time for 6 weeks by the training group. Ankle dorsiflexor training was performed four times a week, two sets per time in the case of females and three sets per time in the case of males for 6 weeks, by another group. Center of pressure sway amplitude was measured using the F-scan system during gait. All subjects were assessed with the same measurements at a pre-study examination and reassessed at eight weeks. Data were analyzed statistically using the paired t-test and one-way ANOVA. [Results] Among the between ankle dorsiflexor training group, static muscle stretching group, and control group, the difference before and after the training were proven to be statistically significant. [Conclusion] Compared to other training groups, the ankle muscle strength training group showed statistically significant increases of forward thrust at stroke patients' toe-off which positively affected stroke patients' ability to perform gait.
\end{abstract}

Key words: Plantar pressure, Stroke, Muscle stretching

(This article was submitted May 16, 2013, and was accepted Jul. 5, 2013)

\section{INTRODUCTION}

The gait pattern of stroke patients is characterized by a slow gait cycle and velocity, a difference in stride lengths between the affected and unaffected sides, and short stance and relatively long swing phases on the affected side ${ }^{1)}$. In particular, when the stiffening of the flexor on the bottom of the ankle joint is severe, it hinders the advance of the lower limbs during gait, resulting in problems such as asymmetric postures, balance disorders, and the loss of ability of motor control in the performance of detailed motor functions ${ }^{2)}$. The gait function relies on the functions of both lower limbs and needs be recovered sufficiently to enable functional activities $^{3)}$. Gait is the function that is first considered when minimizing the disorders of stroke patients and having them return to an independent daily life ${ }^{4)}$. In the process of the functional recovery of stroke patients, improving the gait ability becomes the prime purpose of physical therapy, because gait is an important factor in realizing functional independence ${ }^{5)}$. For functional walking, the foot is the most important element in gait. Walking is an important trans-

*Corresponding author. Jin Hwan Lee (E-mail: poohpt22@, naver.com)

C2013 The Society of Physical Therapy Science

This is an open-access article distributed under the terms of the Creative Commons Attribution Non-Commercial No Derivatives (by-ncnd) License $<$ http://creativecommons.org/licenses/by-nc-nd/3.0/>. port means for humans. The feet not only provide the propulsion and direction necessary for trunk movements, but also play the role of absorbing physical shocks, supporting the body weight, and simultaneously maintaining balance and the stability of the feet themselves by responding to adaptation to the ground and the movement of the center of gravity. The ankle joint, which is important in gait, absorbs shocks during walking, provides a stable bearing surface in a weight bearing posture and enables the advance of the lower limbs. Achache et al. ${ }^{6)}$ stated that the spinal excitability in response to the flexor in the back of the ankle extending to the knee joint extensor particularly increases when stroke patients are walking, and in the early stance phase, the functional recovery of the flexor in the back of the ankle joint influences the improvement in the patients' gait ability by helping to stabilize the knee. Lin $^{7)}$ reported that the movements of the center of plantar pressure on the affected side increased from side to side, but decreased forward and backward, and most of all, improvement in the ability of the ankle joint had a major effect on gait velocity and stride length. The movements of the center of plantar pressure also increased forward and backward. In a study of the gait ability of stroke patients, Adler et al. ${ }^{8)}$ noted that the contract-relax technique, which is a proprioceptive neuromuscular facilitation (PNF) technique, can be applied to cause concentric contraction in limited antagonistic muscles against static resistance, and induce relaxation within the increased range due to the concentric contraction, as 
well as increasing the flexibility of muscles related to the ROM. Stretching exercises to improve the mobility of soft tissues and the ROM are divided into passive and active stretching exercises according to the ways in which they are performed. Regardless of the stretching exercise method, it is important to relax shortened muscles and induce limited connective tissues to stretch easily ${ }^{9)}$. As noted above, various studies have emphasized the importance of flexion in the back of the ankle for the effective gait patterns of stroke patients. Therefore, exercises that can increase the ROM of the ankle joint and strengthen the flexor in the back of the ankle joint are necessary. This study focused on the fact that the undesirable gait exhibited by stroke patients is the result of weakening of the ankle muscles and the lack of their activation. This study implement ankle joint dorsiflexion training for the ankle muscle strength weakness that impairs stroke patients' gait performance to examine the effect of the training on stroke patients' gait and its relationship with C.O.P away amplitude. We conduct muscle strengthening or static muscle stretching training for patients with hemiplegia due to stroke through flexion of the back of the ankle and analyze the C.O.P sway amplitude before and after the intervention, to identify the effects of the respective training programs.

\section{SUBJECTS AND METHODS}

The study subject were 45 patients who had been diagnosed with stroke by computed tomography (CT) or magnetic resonance imaging (MRI), had a Brunnstrom stage higher or equal to stage 3 , met the selection criteria set by the study, and agreed to participate in the study. The subjects were divided into 15 patients who were treated with ankle muscle strength training in addition to conventional physical therapy, 15 patients who were treated with static muscle stretching training in addition to conventional physical therapy, and 15 patients who were treated only with conventional physical therapy. All of them understood the purpose of this study and gave their written informed consent before experimental involvement. The study was performed according to the orinciples of the Declaration of Helsinki, and ethical approval was granted by the local committee of the Institution Review Board of university hospital. This study was conducted with patients hospitalized at K Hospital, Buk-gu, Daegu, in 20-min sessions, four times a week over a six-week period from December 2010 to March 2011.

The control group only received conventional physical therapy that consisted of 20 minutes of ergometric bicycle training, 30 minutes of functional electrical stimulation (FES), and 30 minutes of exercise therapy. The exercise therapy was comprised of weight-shift training, gait training, muscle strength training, and stretching exercises.

The static muscle stretching training group received the same conventional physical therapy as the control group. Then each subject in this test group was instructed to direct the heels downward and the toes upward, and perform muscle stretching for 20 minutes in a standing position using the flexor on the bottom of the ankle joint while keeping the ankle joint posteriorly flexed at $20^{\circ}$ with an inclined board for both feet.

The ankle muscle strength training group received the same conventional physical therapy as the control group. Then each subject lay on a bed and performed posterior flexion of the ankle joint for seven seconds against a resistance force of 20 to $30 \%$ of the individual's maximum muscular strength. Afterward, the therapist created an equal opposite pressure to the flexion of the back of the ankle joint by using his/her hands, to induce isometric contraction in the muscle in front of the shinbone. After the contraction, the subject was given a 10 -second break. The female subjects repeated two sets of the above exercise and the male subjects performed three sets of the above exercise. A 10-second break was provided between sets ${ }^{10,11)}$. As the training intervention progressed, the intensity of the exercises was increased by 40 to $80 \%$.

The sway of C.O.P in the stance phase was measured using the F-Scan system (Tekscan Inc., South Boston, MA, USA). The F-Scan system consists of a pressure sensorarray that can be cut to size, and it measures plantar pressures. A transducer is attached to the legs, and a cable connects the transducer to a computer, which is used to analyze the plantar pressures. The subjects had to walk three times at their usual gait velocity and the average of the three measurements was used in the analysis. The input of signals were delivered to a computer via a $10 \mathrm{~m}$-long cable which was attached to an interface attached to the waist of each subject. The data were collected and analyzed using the software F-Scan research TAM/STAM 6.00 (Tekscan Inc., South Boston, MA, USA).

The data analysis was performed after calculating the averages and standard deviations of the measurements data using SPSS 12.0 for Windows. The general characteristics of the subjects were analyzed through descriptive statistics. In addition, the paired t-test was employed to examine changes in the gait ability of each group before and after the training. In addition, to verify the homogeneity of the three groups of subjects, plantar pressures and gait indices were compared among the three groups, and compare their reciprocity, the data were analyzed using one-way ANOVA. The statistical significance level was chosen as $\alpha=0.05$.

\section{RESULTS}

Forty-five subjects initially participated in this study however, six were discharged from the hospital and three did not participate for personal reasons. As a result, the final experiment involved 36 subjects.

The control group consisted of 12 subjects, seven men and five women., They were (mean \pm SD) $52.08 \pm 3.08$ years of age, $166.75 \pm 2.26 \mathrm{~cm}$ in height, $63.66 \pm 2.20 \mathrm{~kg}$ in weight, and had $12.83 \pm 2.14$ months of disease duration. In terms of the patterns of hemiplegia, seven subjects were affected on the right side and five were affected on the left side. When classified by the causes of the condition, nine people had experienced cerebral infarction and three people had experienced cerebral hemorrhage. The static muscle stretching training group consisted of 12 subjects seven men and 
Table 1. General characteristics of the subjects

\begin{tabular}{lccc}
\hline Variables & $\begin{array}{c}\text { CG } \\
(\mathrm{n}=12)\end{array}$ & $\begin{array}{c}\text { SMSTG } \\
(\mathrm{n}=12)\end{array}$ & $\begin{array}{c}\text { ADFTG } \\
(\mathrm{n}=12)\end{array}$ \\
\hline Gender (male / female, $\mathrm{n})$ & $7 / 5$ & $7 / 5$ & $9 / 3$ \\
Age $(\mathrm{yr})$ & $52.08 \pm 3.08$ & $54.41 \pm 3.23$ & $52.91 \pm 2.96$ \\
Height $(\mathrm{cm})$ & $166.75 \pm 2.26$ & $167.91 \pm 2.87$ & $167.25 \pm 3.61$ \\
Weight $(\mathrm{kg})$ & $63.66 \pm 2.20$ & $64.83 \pm 2.49$ & $67.66 \pm 2.15$ \\
Time since stroke (month) & $12.83 \pm 2.14$ & $15.41 \pm 2.47$ & $12.33 \pm 2.20$ \\
Foot size (mm) & $255.41 \pm 3.45$ & $257.91 \pm 3.91$ & $252.91 \pm 4.01$ \\
Paretic side (right / left, $\mathrm{n})$ & $7 / 5$ & $4 / 8$ & $5 / 7$ \\
Type of stroke (infarction / hemorrhage, $\mathrm{n})$ & $9 / 3$ & $6 / 6$ & $5 / 7$ \\
\hline
\end{tabular}

$(\mathrm{Mean} \pm \mathrm{SE}), \mathrm{ADFTG}=$ Ankle dorsiflexion training group, $\mathrm{SMSTG}=$ Static muscle stretching training group, $\mathrm{CG}=$ Control group

five women. They were (mean \pm SD) $52.41 \pm 3.23$ years of age, $167.91 \pm 2.87 \mathrm{~cm}$ in height, $64.83 \pm 2.49 \mathrm{~kg}$ in weight, and $15.41 \pm 2.47$ months of disease duration. As for the patterns of hemiplegia, four subjects were affected on the right side and eight were affected on the left side. In terms of the causes of the condition, six subjects had experienced cerebral infarction and six had experienced cerebral hemorrhage. The ankle muscle strength training group consisted of 12 subjects, nine men and three women. They were (mean $\pm \mathrm{SD}$ ) $52.91 \pm 2.96$ years of age, $167.25 \pm 3.61 \mathrm{~cm}$ in height, $67.66 \pm 2.15 \mathrm{~kg}$ in weight, and had $12.33 \pm 2.20$ months of disease duration. As for the patterns of hemiplegia, five subjects were affected on the right side and seven were affected on the left side. In terms of the causes of the condition, five had experienced cerebral infarction and seven had experienced cerebral hemorrhage. The homogeneity and normality tests revealed no statistically significant differences among the three groups $(\mathrm{p}>0.05)$ (Table 1$)$.

The paired t-test was conducted to examine differences in C.O.P sway amplitude in the plantar pressure before and after the training during the gait of the control, static muscle stretching, and ankle muscle strength groups. The test results were as follows.

In the control group, the C.O.P sway amplitude was $16.97 \pm 0.81 \mathrm{~cm}$ before the training and $17.68 \pm 0.77 \mathrm{~cm}$ after the training. The comparison of the averages before and after the training revealed an increase in the C.O.P sway amplitued. Moreover, the difference of the averages before and after the training was confirmed to be statistically significant $(\mathrm{p}<0.05)$. In the static muscle stretching training group, the C.O.P sway amplitude were $16.94 \pm 0.83 \mathrm{~cm}$ before the training and $18.23 \pm 0.65 \mathrm{~cm}$ after the training. The comparison of the averages before and after the training revealed an increase in the C.O.P sway amplitede. In addition, the difference of the averages before and after the training was proven to be statistically significant $(p<0.05)$. In the ankle strengthening training group, the C.O.P sway amplitude was $17.17 \pm 0.81 \mathrm{~cm}$ before the training and $18.42 \pm 0.77 \mathrm{~cm}$ after the training. The comparison of the averages before and after the training revealed an increase in the C.O.P sway amplitude. In addition, the difference of the averages before and after the training was confirmed to be statistically significant $(\mathrm{p}<0.05)$ (Table 2$)$.
Table 2. The comparison of C.O.P in each of the groups at (Unit: $\mathrm{cm}$ )

\begin{tabular}{lccc}
\hline Group & \multicolumn{3}{c}{ Mean \pm SE } \\
\hline & Pre & Post & \\
\hline CG & $16.97 \pm 0.81$ & $17.68 \pm 0.77$ & $*$ \\
SMSTG & $16.94 \pm 0.83$ & $18.23 \pm 0.65$ & $*$ \\
ADFTG & $17.17 \pm 0.81$ & $18.42 \pm 0.77$ & $* *$ \\
\hline
\end{tabular}

$* \mathrm{p}<0.05, * * \mathrm{p}<0.01$

To compare the differences C.O.P sway amplitude in the plantar pressures on the affected side between before and after the training during the gait of the control, static muscle stretching, and ankle muscle strengthening groups, oneway ANOVA was performed. No statistically significant difference was revealed before and after training $(\mathrm{p}>0.05)$.

\section{DISCUSSION}

For stroke patients, the weakening of ankle muscle strength is one of the factors that limits their t' functional recovery, and results in decline muscle strength, balance ability, and functional independence ${ }^{12}$. Exercises to enhance the muscle strength of the ankle joint and the ROM can be helpful for recovery of gait ability after the onset of stroke ${ }^{13)}$. Bohannon and Larkin implemented ankle joint stretching exercises to improve gait ability ${ }^{14}$, reported that when hemiplegic patients, who had reduced flexion the back of the ankle due to the shortening of the gastrocnemius, were treated with the technique of standing using a tilting table with a wedge-shaped board installed, increase in the passive flexion at the back of the ankle was observed.

In the study of Fan Gao et al. ${ }^{15)}$, an increase in the ROM was exhibited after stroke patients performed repeated stretching exercises for the ankle joint in a sitting position using a machine,. Moreover, an increase in the length of the Achilles tendon was detected by ultrasonography. In a previous study of muscle strengthening exercises for the ankle joint aiming to improve gait ability, Mattacola and Lloyd ${ }^{16)}$ reported that the application of a proprioceptive exercise program for a six-week period was effective at improving the flexor strength of the back of the ankle. Andrews and 
Bohannon $^{17)}$ performed a study of the short-term recovery of the lower limb muscle strength of stroke patients. They reported that the flexor strength of the back of the ankle on the affected side increased from $75.6 \mathrm{~N}$ at the time of hospitalization to $102.4 \mathrm{~N}$ after the intervention, a statistically significant difference. Docherty et al. ${ }^{18)}$ reported that after a six-week ankle joint exercise program, a statistically significant difference in the flexor strength of the back of the ankle was observed. The present study investigated the effects of ankle joint muscle strength training and static muscle stretching programs on C.O.P sway amplitude. The static muscle stretching group performed static stretching while the ankle joint was flexed $20^{\circ}$ at the back of the ankle on an inclined board. The comparison of the pre- and posttraining results revealed a statistically significant increase in C.O.P sway amplitude. We consider the statistically significant difference in the C.O.P sway amplitude between before and after the training found in this study was the result of reduced stiffening and increased ROM, which were also observed in the previous studies that conducted stretching exercises. In the training group in which muscle strengthening exercises were applied to the flexor in the back of the ankle joint, the C.O.P sway amplitude increased after the training. As with the results of the previous studies that conducted muscle strengthening exercises, the functional improvement in the flexor at the back of the ankle may have enabled the subjects to accurately touch their heels on the ground in the stance phase and increase the contact area of the foot. This would have provided the ankle joint with stability against the bearing surface during gait and helped stroke patients to effectively walk on their own, thereby having an overall positive influence on gait ability. The muscle strengthening exercises used in the present study followed the technique that Chaitow mostly used in the field of orthopedic physiotherapy. They employ a reciprocal inhibition technique using a protocol in which the antagonistic muscle of the respective muscle is inhibited in isometric contraction, and then immediately relaxed. Therefore, the exercises are considered as a treatment method within the concept of therapeutic exercise, 'relaxation and facilitation'. The contract-relax technique, which is one of proprioceptive neuromuscular facilitation techniques that are frequently used for stroke patients, may partly overlap with the method used to increase ROM-related muscular flexibility and muscular strength using concentric contraction against static resistance and inducing the relaxation of the antagonistic muscle within the range increased by the concentric contraction. Given the results of the present study, it can be seen that ankle joint dorsi flexion training implemented in addition to conventional physical therapy increased stability during gait by increasing C.O.P sway amplitude during gait. Compared to other training groups, the ankle muscle strength training group showed statistically significant increases in sway amplitude, and it can be said that this training increases forward thrust at stroke patients' toe-off positively affecting stroke patients' ability to perform gait. The present study measured the C.O.P sway amplitude as a variable of the gait of stroke patients, using the F-Scan system.
This system was developed in the USA, and it measures plantar pressures and partly measures gait characteristics. It is capable of analyzing various gait patterns during walking and senses pressures in various regions of the foot. In addition, it has the advantage of providing temporal and quantitative data. The F-Scan system is known to provide more practical data for measuring the ground reaction force than a force plate and has a high level of reliability ${ }^{19}$. However, it has the limitation of being unable to analyze qualitative elements and compensatory actions of gait. The limitations of this study were that the study findings cannot be generalized all stroke patients given the small number of subjects in each group, and the control group had a shortage of training time, to some extent, compared to the test group.

\section{REFERENCES}

1) Mauritz KH: Gait training in hemiplegia. Eur J Neurol, 2002, 9 Suppl 1: 23-29; dicussion 53-61.

2) Caillet F, Mertens $P$, Rabaseda $S$, et al.: Three dimensional gait analysis and controlling spastic foot on stroke patients. Ann Readapt Med Phys, 2003, 46: 119-131. [Medline] [CrossRef]

3) Kollen B, van de Port I, Lindeman E, et al.: Predicting improvement in gait after stroke: a longitudinal prospective study. Stroke, 2005, 36: $2676-$ 2680. [Medline] [CrossRef]

4) Patrick JH, Keenan MA: Gait analysis to assist walking after stroke. Lancet, 2007, 369: 256-257. [Medline] [CrossRef]

5) Turnbull GI, Charteris J, Wall JC: A comparison of the range of walking speeds between normal and hemiplegic subjects. Scand J Rehabil Med, 1995, 27: 175-182. [Medline]

6) Achache V, Mazevet D, Iglesias C, et al.: Enhanced spinal excitation from ankle flexors to knee extensors during walking in stroke patients. Clin Neurophysiol, 2010, 121: 930-938. [Medline] [CrossRef]

7) Lin SI: Motor function and joint position sense in relation to gait performance in chronic stroke patients. Arch Phys Med Rehabil, 2005, 86: $197-$ 203. [Medline] [CrossRef]

8) Adler SS, Beckers D, Buck M: PNF in practice. An illustrated guide, 3rd ed. Berlin: Springer-Verlag. 2008, 3: pp 78-82.

9) Kisner C, Colby LA: Therapeutic exercise. Foundations and techiques. 4th ed. Philadelphia: F A Davis, 2002, pp 34-61.

10) Dean CM, Richards CL, Malouin F: Task-related circuit training improves performance of locomotor tasks in chronic stroke: a randomized, controlled pilot trial. Arch Phys Med Rehabil, 2000, 81: 409-417. [Medline] [CrossRef]

11) Weiss A, Suzuki T, Bean J, et al.: High intensity strength training improves strength and functional performance after stroke. Am J Phys Med Rehabil, 2000, 79: 369-376; quiz 391-364

12) Whitney SL, Poole JL, Cass SP: A review of balance instruments for older adults. Am J Occup Ther, 1998, 52: 666-671. [Medline] [CrossRef]

13) Runge CF, Shupert CL, Horak FB, et al.: Ankle and hip postural strategies defined by joint torques. Gait Posture, 1999, 10: 161-170. [Medline] [CrossRef]

14) Bohannon RW: Device for increasing passive ankle dorsiflexion at home. Suggestion from the field. Phys Ther, 1985, 65: 1521. [Medline]

15) Gao F, Ren Y, Roth EJ, et al.: Effects of repeated ankle stretching on calf muscle-tendon and ankle biomechanical properties in stroke survivors. Clin Biomech (Bristol, Avon), 2011, 26: 516-522. [Medline] [CrossRef]

16) Mattacola CG, Lloyd JW: Effects of a 6-week strength and proprioception training program on measures of dynamic balance: a single-case design. $\mathrm{J}$ Athl Train, 1997, 32: 127-135. [Medline]

17) Andrews AW, Bohannon RW: Short-term recovery of limb muscle strength after acute stroke. Arch Phys Med Rehabil, 2003, 84: 125-130. [Medline] [CrossRef]

18) Docherty CL, Arnold BL, Zinder SM, et al.: Relationship between two proprioceptive measures and stiffness at the ankle. J Electromyogr Kinesiol, 2004, 14: 317-324. [Medline] [CrossRef]

19) Catalfamo $P$, Moser $D$, Ghoussayni $S$, et al.: Detection of gait events using an F-Scan in-shoe pressure measurement system. Gait Posture, 2008, 28 420-426. [Medline] [CrossRef] 